\title{
Design Considerations for Capacity in PRT Networks
}

\author{
Jörgen Gustafsson ${ }^{1}$, Jong-Gu Kang ${ }^{2}$, Johan Englund ${ }^{3}$ and Peter Grimtell ${ }^{4}$
}

${ }^{1}$ Chief Technical Officer, Vectus Ltd, Husargatan 2, S-752 37 Uppsala, Sweden; jorgen.gustafsson@vectusprt.com

${ }^{2}$ Traffic Systems Engineering, Vectus Ltd, Mosan Building 5:th Floor, 14-4 Yangjaedong Seocho-gu, Seoul, 137-888, South Korea, joonggu.kang@ vectusprt.com

${ }^{3}$ Senior Developer, Noventus Systems AB, Gyllenkroksgatan 10B, 41261

Gothenburg, Sweden, johan.englund@noventus.se

${ }^{4}$ Senior Developer, Noventus Systems AB, Gyllenkroksgatan 10B, 41261

Gothenburg, Sweden, peter.grimtell@ noventus.se

\section{ABSTRACT}

There has been increasing interest in automated networks using small vehicles during the last several years. Such systems can provide a high level of passenger service with a comparably lower investment, potentially making them an attractive alternative to other technologies such as people-movers, monorails, etc. The subject matter of this discourse expands upon design considerations relating to capacity for such automated small vehicle systems, often called Personal Rapid Transit (PRT). Vectus has operated a test track with three vehicles in Uppsala, Sweden, since 2007. To verify performance in a larger system, an emulator was developed which runs the actual safety-validated control software including all communications and the complete operator interface. The hardware in the system, vehicles, propulsion, platform doors, ticketing, etc., are emulated with very detailed and calibrated software models. In this way several uncertainties for accuracy and real-life representation associated with the use of simulations is removed. The emulations show that high capacity can be achieved, but more importantly, there are several other factors (other than the control system) that have a significant impact on performance. It is imperative that all factors are properly considered in the system design in order to achieve high capacity - factors that many simulations may not account for in an accurate way.

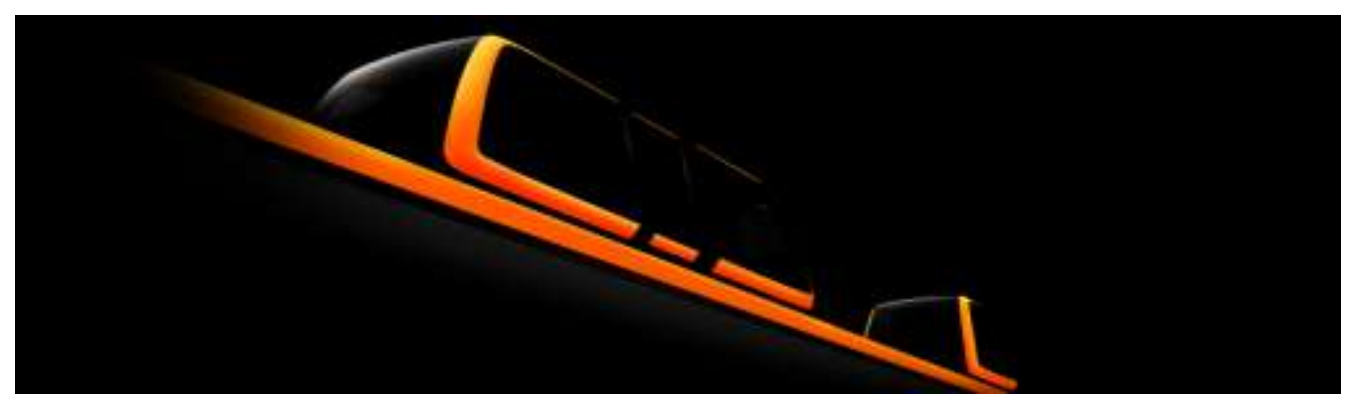

Figure 1: Design teaser of Vectus" "next-generation" large and small vehicle. 


\section{INTRODUCTION}

Vectus has operated a test track with three vehicles in Uppsala, Sweden, since 2007. The control system has been a key component of the system from the very beginning and one of the key purposes of the test track was to verify the control system. The specification for the control system called for the functionality that is widely recognized as being required for a high flow of vehicles. This includes short headways, asynchronous control, and moving block technology (and linear stations). The control system has undergone extensive testing at the test track. The control system's safety case has been subjected to a third party analysis and is approved by the relevant authorities based on requirements for generic applications.

Since the test track's early experiences in late 2008 [2], there has been a period of gaining further experience enhanced by almost continuous operation and many visitors. Today more than 2000 visitors have experienced the system, and several thousand hours of vehicle operation have been amassed. This has provided valuable feedback and an increased understanding of the operational properties of the automated system. Tests have also successfully demonstrated merging at full speed while maintaining short headways, speeds over $50 \mathrm{~km} / \mathrm{h}$, and towing and pushing for rescue and recovery purposes. The test track uses in-track linear motor propulsion [1], which has been tested and proven successful in severe snow and ice conditions for several consecutive winters. On-board propulsion, linear motors, or tire drive, are used when climate conditions permit.

Visitors, both transportation professionals as well as laymen, have obtained a quick and clear understanding of the advantages and features of the system from a passenger point of view. It has, however, been more difficult to convey the system's ability to perform at a high enough level of service to manage peak-hour requirements in typical applications without very detailed discussions.

There has been a growing interest in PRT systems from both cities as well as private entities. Increasing numbers of consultants have presented studies and simulations, claiming specific passenger capacities and required number of vehicles for the applications they have studied. Headways and subsequent line capacities are usually the only topic for discussion and the basis for evaluating a control system's feasibility.

The more simulations for different projects that were seen, the more suggested layouts and station configurations that were proposed, the more concerns Vectus had about the viability of these proposals from consultants and others. Many simulations claimed certain total capacities, but rarely was the exact demand matrix disclosed, or the control and safety methodology. 
In all the different studies conducted, what were the assumptions for the simulations? How are the different systems that are available on the market reflected?

With the commercial systems currently available for automated networks, it is indisputable that the various systems perform differently in various conditions. Station layout is one example, where different layouts each have their pros and cons. In terms of capacity for high vehicle (and passenger) throughput, the differences are significant [3].

If a client decides to proceed with procurement based on a certain layout and a given number of vehicles, will the expected system transportation performance be achieved or vice versa, would a cheaper system solution have sufficed? Vital aspects are seldom properly discussed, such as performance in case of various failures, or behavior in overload situations. The question that has to be asked is if these so-called experts have the full picture.

This triggered a need to reconfirm that the actual software that was developed for the system, (with the safety segment of the software certified and finalized), actually performed in the larger system and could provide the overall capacity requested. Was Vectus' reality better or worse than the more or less fictive simulators used by various consultants.

The solution for finding answers to all these questions was to build a large system with all systems properly represented in the smallest detail - a "real-time" system that used all computers, all software, all communications and which replicated the exact behavior of all parts of the system (vehicles, etc.). It was only in this manner that a true and undisputable verification for the larger system performance could be achieved.

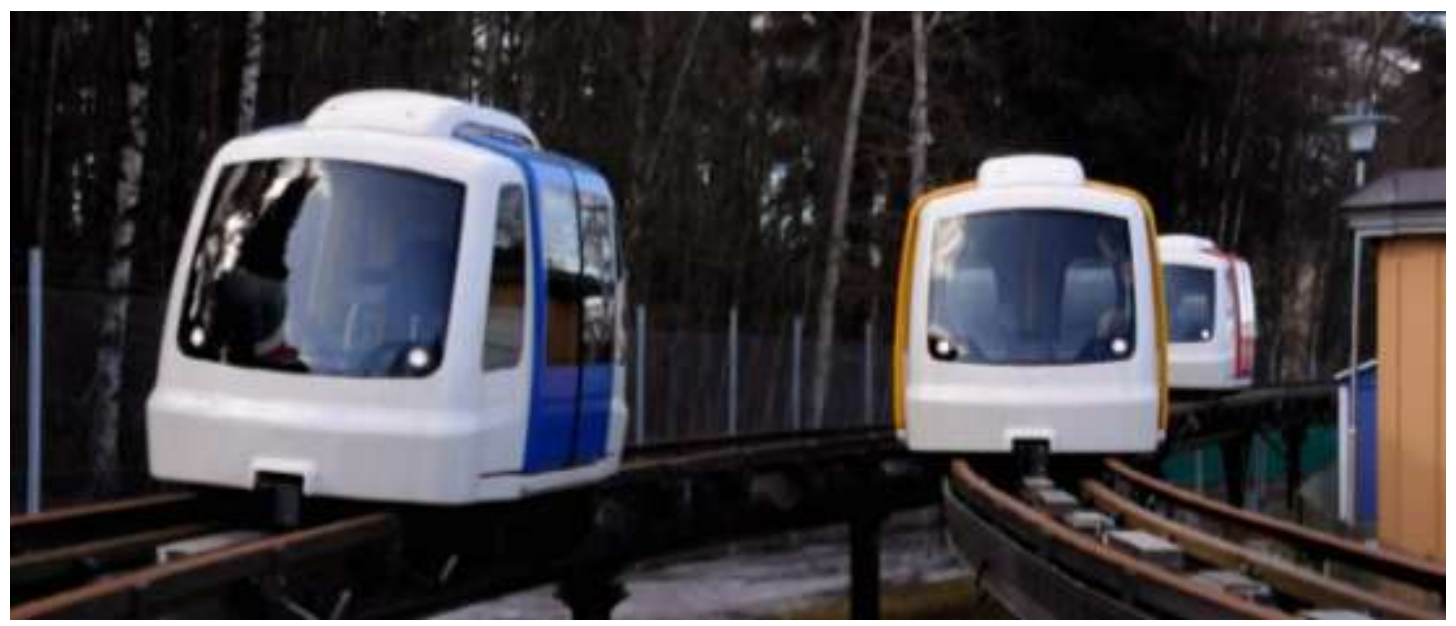

Figure 2. Diverge at test track, spring 2009 
In the next section of this discourse, the following subjects will be discussed: the development of the emulator, the results achieved, and the conclusions made regarding the capacity for small vehicle networks (PRT).

\section{VECTUS CONTROL SYSTEM}

Prior to the test track being built, various studies had been conducted over several years. One important aspect of these studies was the overall control and the logistics solutions of a real system, which were studied using advanced simulation tools. Based on these studies, the key parameters for a commercially viable system were identified.

The results from this early analysis are summarized here for the purpose of clarity.

\section{Distributed and scalable control}

A distributed system means that the control is carried out locally for a limited part of the system. With the distributed system there is no increase in the load for each individual control segment when the system is expanded.

\section{Asynchronous Control}

With asynchronous control the flow of vehicles is handled as they travel along their path to their destinations. Merging of vehicles is managed as required on a local basis. Occasionally there may be a need to slow down in order to facilitate merging in switches. Travel time may be prolonged by a few seconds, but the overall capacity of the system is maintained.

\section{Vehicle Spacing - Dynamic Moving Block}

With a dynamic moving block, the distance between the vehicles can be varied depending on the speed of the vehicle (or rather the actual stopping distance, depending on the speed). Optimal vehicle flow can be maintained at different speeds on a given track section. At low speeds, e.g. in stations, this feature easily allows vehicles to move and park, with very small distances between them.

\section{Optimal Control.}

The above systems are the building blocks in providing safety as well as adequate capacity. A higher level of system control is required for managing the logistics of vehicle flow. Empty vehicle management and dynamic route selection are examples of what are seen as important features. 


\section{THE EMULATOR}

The Vectus control system involves several computers. Each vehicle has a vehicle control computer managing the entire vehicle's functionality. There is also a Safety Vehicle Controller monitoring and ensuring the safety aspects of the vehicle. There are multiple communication paths between the vehicle and the infrastructure. In the infrastructure there are distributed controllers and safety controllers along the track (similar to ATC fixed installations). Each station has a set of controllers, as do platform doors, inverters for linear motors, ticketing machines, platform and vehicle displays, and passenger communications systems.

The complete structure of all processing power is built up in the emulator exactly as required for the real system. This includes running all the correct software for safety systems, operator interface, vehicles, etc., as individual processes, and communicating with each other exactly as the real application would have done. All hardware, i.e. vehicles, actual platform doors, and ticketing systems are emulated. This means that there is a computer program that "emulates" the behavior of the hardware as accurately as possible by means of interfacing with all the IO (digital and analog inputs and outputs, speed sensors, etc.) for each process in the system. The accuracy of the emulation has been verified with the actual performance at the test track. All systems are included in the emulation, so all logs, reports and operator interfaces are live and can also be used in the emulator.

The emulator will also be a useful tool in real applications. New software can be tested and verified before being introduced into the real system. Scenario planning can be done: for example, how will the system perform if this failure occurs, if this specific passenger demand occurs, if the operator resolves a certain situation in this or that way? Operator training in general is another important area. The list of uses for the emulator is long.

Emulator add-ons can be used to model electrical systems using the exact topology for the specific system. This can be used to determine the exact currents and loads in all parts of the electrical system all the way back to the supplying grid.

In a similar way, exact locations of WLAN and radio communications equipment can be entered, and the load can be analyzed in detail for the specific scenario and compared to the overall capability for a given communication system configuration [4]. For the test track only short range communications are used, but for longer range communications solutions, this will be a very important area to validate in order to avoid disruptions during localized high system loads.

The results from emulations can be represented in several different ways. The raw data that is stored contains all relevant data for all vehicles and all transports 
(passengers) for an unlimited emulation time. Typically this is extracted into spreadsheet files. Operational data for each transport can be information such as origin, destination, actual (real) time, waiting time, and travel time. This can then be further processed and analyzed. In a similar way, on a vehicle basis, there is data for each trip origin, destination, number of passengers, distance traveled, idle time, etc. It is always possible to go back to the raw data and analyze the exact circumstances for a particular event.

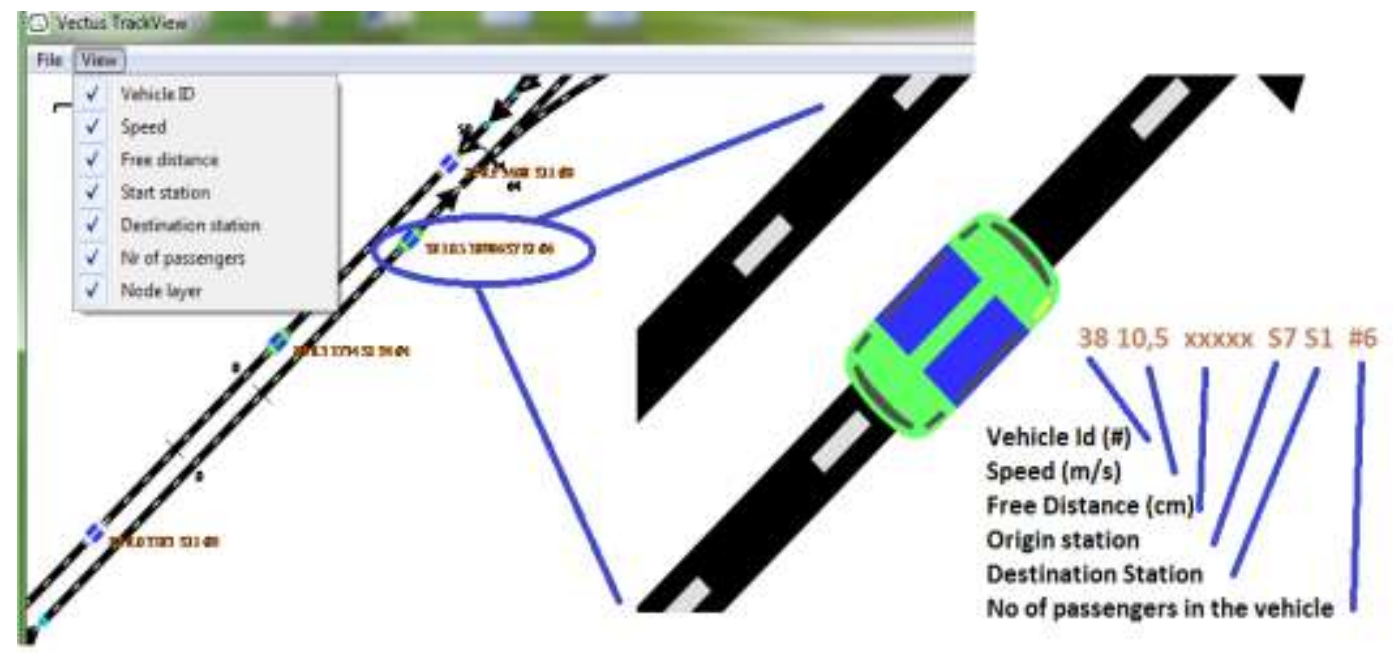

Figure 3. Part of a PRT system as seen in the emulator. A capture tool can be used to record the emulation allowing later playback. Each vehicle has selectable data that can be displayed next to the vehicle to support evaluation during playback.

\section{CAPACITY EXPERIENCE FOR A SMALL VEHICLE SYSTEM}

The first application where the emulator was used was for a proposed network in Uppsala. After programming the system as represented in the feasibility studies, defining all vehicles and entering the same demand matrix with all the individual travelers for each station, their destinations and times for their "ticket" purchase, the first results were achieved. The results were alarming. The passengers using the system during peak hours would have had a difficult experience, and the last passengers would have arrived at their destination with a delay of several hours.

Some problems that were discovered were easy to improve. Track routing was modified in some areas. Sizing of the stations to better correspond with the required throughput of vehicles (not only a matter of number of passengers) also improved performance. Eventually the system performed significantly better than the feasibility study simulation. Several factors had considerable impact on performance. Some of the findings proved what was already suspected. Some findings are obvious in hindsight. The overall lesson is that without the emulator it is virtually impossible to 
design a system properly. Without an emulator, any capacity prediction will contain a large portion of guess-work. Some observations are listed below (not in any particular order):

- Response time for empty vehicles to transfer to locations where there is a need and activation of idle vehicles was sometimes not fast enough. Sometimes after an empty vehicle had been requested, another transport with passengers was generated going to the same station. If the distance was shorter for the new transport, the transport with passengers would arrive earlier, possibly rendering the requested empty vehicle void upon arrival. Prediction methodology as well as continuous updating of needs and rerouting (and cancellation) of empty vehicles improved the situation.

- It is very important to have enough capacity for vehicles to wait at the entrance to a station for berths to become available. If there is no space available, the queue may either block the main line, or require the vehicles to make an extra lap and come back and try again. This applies for both empty vehicles as well as vehicles with passengers. If occasional vehicles are still forced to make an extra lap, there should be a mechanism to prioritize station access the next time the vehicle returns. In the first emulation, some vehicles had the misfortune of making three and four extra laps.

- In this particular application there were three stations that had a (very) high percentage of the total travel in the system. The overall waiting and travel time in the system benefitted significantly from maximizing ride sharing. By simply letting passengers to these destinations wait until the vehicle was full (a maximum wait time was applied) and possibly letting other passengers entering the station later, but who were going to other destinations, depart ahead gave better results overall. Perhaps $30-60$ seconds extra waiting time for traveling the high density route could save several minutes waiting time for worst-case traveler at the less travelled stations. In a sense, this is not far from applying a timetable operation for the high density destinations during peak hour.

- Using the logic from the point above, increasing seating capacity from four to six passengers made a tremendous impact on the station load due to the fact that fewer vehicles were required during peak hour operation. This also helped reduce travel time (less queuing time in the waiting area when arriving at the station).

- With the high load stations being quite some distance apart, the operating speed also made a difference (the system always adjusts speeds in curves to ensure passenger comfort). Rather obviously, the quicker the trip can be made, not only is travel time shortened for the passengers, but each vehicle is 
able to perform more roundtrips and to transport more passengers in a given time frame.

- Ramps at the entrance to the station to allow for braking without impacting the flow of vehicles at the main line was not found to be very important in this application. The same thing applied to acceleration ramps when entering the main line. It is a contradiction in terms to have a very high load station combined with a very high load on the passing main line. The space required for retardation and acceleration ramps are much better used for waiting areas for entering and exiting the station.

- The sequence at the station had a great impact on efficiency. One such sequence that was shown at the test track to be inefficient and subsequently discarded was the sequence where there was first a stop where passengers exited the vehicle and then the car moved forward to another berth where new passengers could enter. Allowing passengers to both exit and enter at the same station berth provided a much better flow of vehicles. The most efficient situation would be the use of a cross-flow, with passengers exiting to one side, and entering from the other side. Planning for vehicles to sometimes wait a short time and then to let the last berth free-up rather than just stop at the first available berth, increased overall flow. With a high flow of vehicles, a large enough waiting area is required and a more or less time-table operation of the station is also required. An efficient flow would be as follows: moving a set of vehicles (platooning) to the station berths, opening the doors, allowing passengers to exit and the next set of passengers to enter, letting the vehicles depart (platooning), and then immediately moving up the next set of vehicles. (This kind of flow is analyzed in [3]).

- One of the PRT stations in the emulation was located at a railway station. The capability of handling a sudden large influx of passengers was also evaluated. This was achieved by emulating the system with 50 passengers (with destination distribution similar to the overall demand matrix) arriving at the station within a three minute time frame. The first trials demonstrated that the time for empty vehicles to move to the station took too long. By adding a small storage area (a 75-meter parallel track) where empty vehicles could wait, the problem was solved. By moving $10-15$ vehicles to the PRT station's waiting area just before the train arrived, and also increasing the number of berths at the station, all 50 passengers had departed from the station with less than five minutes waiting time.

To summarize, the capacity of the system was not limited in any way by the performance of the system's moving of vehicles along the tracks. There were no system limitations such as a lack of computational power or communications overload. Headway was sufficient, and the asynchronous control provided smooth 
movement along the journey. The dynamic moving block provided space-efficient operation, including performance in the station areas. It was also concluded that increasing the number of vehicles beyond a certain limit gave very little extra capacity. The reason for this was that it was the stations that were the bottleneck. Additional vehicles only increased the congestion at the stations. With the adjustments of the station controls as mentioned above, the flow at the stations was increased significantly, and good performance was attained. Perhaps most importantly, at least for the typical size systems in most current studies, it is the stations that are the key for high capacity. Another very high load emulation snapshot is shown in figure 4 . It is clear that during very high load situations, a large portion of the vehicles are at the stations rather than moving about in the system.

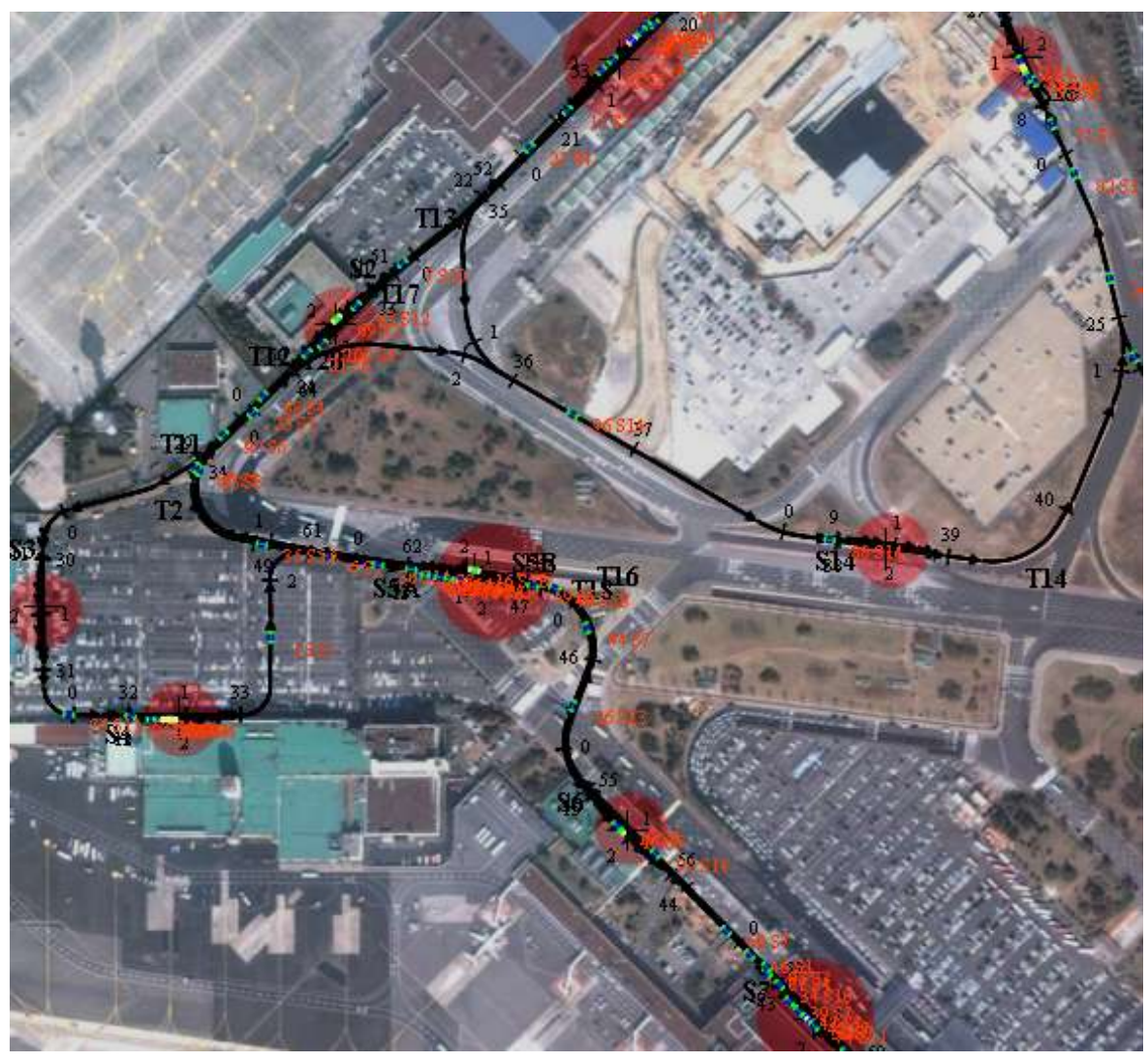

Figure 4. From the density of the vehicles' ID data in this particular view in the emulation, the locations of the stations are easily seen (indicated by the large dots). This is from an $8 \mathrm{~km}$ system with more than 100 PRT vehicles transporting 3500 pph.

The emulator data logs combined with the possibility of visually looking at the vehicles moving along the route provides an invaluable tool to properly understand and analyze system behavior in different conditions. 


\section{CONCLUSIONS}

The first conclusion is that the control system as initially analyzed was mainly concerned with efficient flow of vehicles along the track - to provide transport between destinations. Asynchronous control, dynamic moving block, and distributed control proved more than sufficient for achieving this.

The second conclusion is that different stations require different and much more elaborate operating strategies. It was determined to be very helpful to allow the stations where vehicles arrive to have full control of all transports arriving at the station. This allows for effective planning strategies, both for vehicles arriving at the stations, as well as predicting the passenger (vehicle) flow departing from the stations. This could be viewed as a "station-centered" control concept. Depending upon the operational conditions, the layout and the configuration of the station, individual control schemes for each station may be required.

The third conclusion is based upon the observation that peak hour operation functioned significantly smoother when using the larger vehicle having six seats. The idea of using even larger vehicles circulating between the high load stations is an obvious next step -not so much for the purpose of freeing up the track, but to make station throughput higher, thereby allowing for higher capacity in the system during peak hours.

\section{REFERENCES}

[1] VECTUS - Intelligent Transport: Gustafsson J. Proceedings of the IEEE, Volume 97, Issue 11, Nov 2009.

[2] Vectus PRT Concept and Test Track Experience. Gustafsson J and Lennartsson S. APM 2009 Conference Proceedings.

[3] Towards a PRT Capacity Manual. Joerg Schweizer et al. PRT\&LHR conference 21-23 September 2010.

[4] Determining suitability of the IEEE1609 standard for PRT systems. Johan Englund, University of Gävle, Sweden, 2010. 